\title{
Una traccia machiavelliana nelle pagine del Quijote
}

\section{Walter Ghia}

Università degli Studi del Molise

walter.ghia@unimol.it

\begin{abstract}
Tra le pieghe dello straordinario percorso del Quijote si trova un passaggio la cui ascendenza risale a Machiavelli. C'è poi da riflettere sull'origine di quelle righe divertite e incisive, per cercar di capire se Cervantes abbia proprio letto le pagine del Principe, oppure se sia pervenuto per via indiretta alle idee che lì espone. Appunto per misurare il grado di curiosità e di competenza da parte di Cervantes in fatto di lingua e di scrittori italiani, si getta qui uno sguardo sulla sua familiarità con il filone irriverente e burlesco della letteratura "toscana» del '500. D'altro canto, la traccia machiavelliana che si trova nel Quijote, documenta l'interesse per Machiavelli e per le sue idee, ma non fa di Cervantes un «machiavellico". Nemmeno si può dire che egli sia orientato contro le idee del Fiorentino in virtù di un'opposizione morale o teologica. La distanza tra i due nasce piuttosto dal fatto che Cervantes - come Montaigne- è tra i grandi fondatori di una nuova dimensione del moderno, che si lascia alle spalle le certezze antropocentriche del Rinascimento, mentre fa emergere una diversa immagine dell'uomo: si fa strada infatti, fino ad occupare il primo piano, l'interiorità della dimensione privata, secondo una prospettiva ben diversa da quel primato dell'uomo pubblico che è nota dominante nelle opere machiavelliane.
\end{abstract}

Parole chiave: Machiavelli, Cervantes, Montaigne, primato dell'uomo pubblico, nascita del privato.

\footnotetext{
Abstract

Between the lines, of the amazing literary work Quijote, we find a passage that brings to mind Machiavelli. In these entertaining and incisive lines, there is quite a bit of food for thought to make us reflect whether Cervantes had actually read pages from the Prince or if his ideas had come to him through other means. Therefore, in order to measure Cervantes's degree of curiosity and competence regarding Italian and Italian writers, in this paper we take a glance at Cervantes's familiarity with the irreverent and burlesque line of thought in Tuscan literature belonging to the 1500s. The traces and hints of familiarity with Machiavelli found in Quijote document Cervantes's interest in the Italian writer and his ideas, however these points do not make Cervantes a Machiavellian. In any case, we can not affirm that Cervantes was oriented against the Florentine's ideas considering his moral and theological beliefs. The difference between the two arises from the fact that Cervantes as Montaigne is among the founding fathers of a new dimension of Modern man, that leaves behind the anthropocentric certainties of the Renaissance, and promotes the emergence
} 
of a different image of Man, which arises from the interiority of the private dimension, an outlook in stark opposition to the Public Figure known as the dominant theme in Machiavelli's works.

Key words: Machiavelli, Cervantes, Montaigne, primacy of the Public Man, beginning of the Private Dimension.

Non occorre neppure andare molto avanti nella lettura. Siamo nella prima parte, al capitolo xv, Donde se da cuenta de la desgraciada aventura que se topó don Quijote al topar con unos desalmados yangueses. Rocinante - nonostante l'età, la magrezza e le molte disavventure - si allontana dallo spazio dove Quijote e Sancho stanno mangiando e bevendo: il suo olfatto è buono, ed egli aspira a soddisfare i suoi desideri di maschio con alcune cavalline galleghe che pascolano nella radura. Viene respinto dalle cavalline, e poi abbattuto al suolo a bastonate dai carrettieri di Yanguas. Quijote è indignato e si adopera a vendicare l'offesa nonostante il gran numero di avversari. Per farlo trascura persino le differenze di rango: sappiamo tutti che lui e Sancho ne ricavano una gran dose di legnate.

I due non si sono ancora rialzati, quando Quijote, allarmato dai propositi di acquiescenza e di remissività del suo dolorante scudiero, ritorna sulla promessa di renderlo governatore di un'isola. Nell'argomentazione che viene sviluppata di seguito mi sembra difficile non cogliere anche più di una eco dell'incipit del più famoso tra i capitoli del Principe, il «De principatibus novis qui alienis armis et fortuna acquiruntur»:

Coloro e quali solamente per fortuna diventano, di privati, principi, con poca fatica diventono, ma con assai si mantengano; e non hanno alcuna difficultà fra via, perché vi volano; ma tutte le difficultà nascono quando e' sono posti. E questi tali sono quando è concesso ad alcuno uno stato o per denari o per grazia di chi lo concede [...] Questi stanno semplicemente in sulla volontà e fortuna di chi lo ha concesso loro, che sono dua cose volubilissime et instabili. E non sanno e non possono tenere quello grado: non sanno, perchè se non è omo di grande ingegno e virtù, non è ragionevole che, sendo vixuto sempre in privata fortuna sappia comandare; non possono perchè non hanno forze che gli possino essere amiche e fedele. Dipoi gli stati che vengano subito [...] non possano avere le barbe e corrispondenzie loro: in modo che il primo tempo avverso le spenga $[. . .]^{1}$

Ven acá pecador; si el viento de la fortuna, hasta ahora tan contrario, en nuestro favor se vuelve, llevándonos las velas del deseo para que seguramente y sin contraste alguno tomemos puerto en alguna de las ínsulas que te tengo prometida, ¿qué sería de tí si, ganandola yo, te hiciese señor della? Pues, ¿̇lo vendrás a imposibilitar por no ser caballero, ni quererlo ser, ni tener valor ni intención de vengar tus injurias y defender tu señorío? Porque has de saber que en los reinos y provincias nuevamente conquistados nunca están tan quietos los áni-

1. Niccolò Machiavelli, Opere, vol. I , t. I, a cura di R. Rinaldi, Torino: Utet, 1999, p. 171. 
mos de sus naturales, ni tan de parte del nuevo señor que no se tengan temor de que han de hacer alguna novedad para alterar de nuevo las cosas, y volver, como dicen a probar ventura; y asì es menester que el nuevo posesor tenga entendimiento para saberse gobernar, y valor para ofender y defenderse en qualquiera acontecimiento. ${ }^{2}$ [corsivo mio]

Forse, alcune righe centrali del passo cervantino — quelle che insistono sul fatto che i sudditi del principato nuovo, essendo già stati necessariamente spettatori o protagonisti di una "novità", non temono l'innovazione e hanno la disponibilità mentale ad assecondarne un'altra («no se tengan temor de que han de hacer alguna novedad para alterar de nuevo las cosas») — hanno anche maggiore affinità con la conclusione del capitolo II del Principe: quello che spiega le ragioni della stabilità del principato ereditario, per il quale vale la considerazione che «nella antiquità e continuità del dominio sono spente le memorie e le cagioni delle innovazioni», laddove invece per il dominio recentemente acquisito vale il principio che «sempre una recente acquisizione lascia lo addentellato per la aedificazione dell'altra». ${ }^{3}$

Non si tratta di un passaggio che fa pensare a Machiavelli per analogie e suggestioni percepite secondo un angolo prospettico molto ampio. Non è qui il caso di chiamare in causa l'antropologia machiavelliana. Nel passo, anche i temi della virtù e della fortuna sono presenti in forma discreta, in stretto riferimento alla circostanza rappresentata. Intendo dire che non stiamo calcando terreni magari profondi e affascinanti, ma anche scivolosi e sfuggenti al controllo. Al contrario, la categoria del principato nuovo, acquisito per grazia di altri e non per propria virtù, è in Machiavelli una categoria "tecnica» che occupa il centro della sua analitica dei modi del conseguimento del potere e delle implicazioni che ne derivano per il successivo governo dello stato. $\mathrm{E}$ a dire la verità, proprio per questo, mi pare difficile leggere quel passaggio di Cervantes senza coglierne l'origine in Machiavelli. Anche i modi del porgere nell'argomentare di Quijote hanno la loro importanza. Certamente è una forma di sapienza non occasionale quella che viene qui chiamata in causa dal protagonista della novela. Infatti Quijote assume il tono didascalico di chi si esprime fondandosi su un sapere dottrinale, su una teoria ben precisa. Il passo è introdotto da una premessa che annuncia la correzione di un errore a partire da una dottrina - "Quisiera tener aliento [...] para darte a entender, Panza, en el error en que estás»- e rafforza ulteriormente il suo registro dotto intercalando l'espressione "porque has de saber [...]». Senza dire che insieme a una puntuale aderenza sul piano logico e contenutistico si constata anche una precisa corrispondenza tra alcune parole-chiave: «ingegno e virtù» scrive il Fiorentino, «entendimiento [...] y valor» troviamo in Cervantes.

2. Miguel de Cervantes, Don Quijote de la Mancha, Edición del Instituto Cervantes 16052005, dirigida por Francisco Rico, Centro para la edición de los clásicos españoles, Barcelona: Galaxia Gutenberg, 2004, p. 177.

3. Niccolò Machiavelli, Opere, vol. I, t. I, cit., p. 117. 
Per quanto ho letto non ho mai trovato richiamata l'ascendenza machiavelliana di quel passo negli apparati critici di edizioni del Quijote e neppure in saggi di critica letteraria. La stessa accuratissima e monumentale edizione diretta da Francisco Rico non reca a tal proposito alcun riferimento, neppure attraverso la straordinaria ricchezza di rinvii del volumen complementario. ${ }^{4}$ Eppure non sono né l'unico né il primo ad affermare quel nesso. Infatti, un discreto lavoro di ricognizione $\mathrm{mi}$ ha condotto a verificare che tale ascendenza machiavelliana non era sfuggita al padre illustre della storia delle idee politiche nella Spagna del novecento. José Antonio Maravall nell'ultimo capitolo di Utopía y contrautopía en el Quijote (1976) citava il passo cervantino come raro esempio di una visione dell'arte di governo eccentrica rispetto all'opera, e destinata ad essere sommersa dal contesto: "que va desapareciendo a lo largo del libro». Poi, nella corrispondente nota a pie’ di pagina commentava: «Este planteamiento de las condiciones que ofrece un señorio nuevo procede, aunque sin duda por via indirecta, de Il Principe». ${ }^{5}$ Proprio nel medesimo saggio, Maravall aveva tenuto a sottolineare poche pagine addietro che «ni una sola vez aparece en la larga lista de los autores citados a través de sus abundantes páginas, que Angel Valbuena pacientemente ha confeccionado, el nombre de Maquiavelo». ${ }^{6}$ Cito entrambi i passi perchè messi l'uno accanto all'altro sono forse rivelatori di un qualche imbarazzo. Da un lato lo storico Maravall tiene a sottolineare l'assenza di Machiavelli come una conferma funzionale alla sua interpretazione del Quijote, ${ }^{7}$ dall'altro lato il filologo Maravall non può fare a meno di annusare l'odore di Machiavelli. Quella nota ha più il carattere di una concessione necessaria alla probità scientifica, che non quello della scoperta di un dato che sollecita a sua volta qualche interrogativo.

Sul percorso compiuto da quella categoria delle configurazioni politiche — che ha la sua prima origine nel testo machiavelliano, e giunge fino alla pagina del Quijote - merita spendere qualche nota di riflessione.

4. Nell'edizione diretta da Rico, Machiavelli è citato una sola volta in una nota al capitolo XLVII della prima parte, per commentare l'uso della parola virtud da parte di Cervantes. Cfr. ed. cit., p. 592, n.13.

5. José Antonio Maravall, Utopía y contrautopia en el Quijote, Santiago de Compostela: Editorial Pico Sacro, 1976. Cfr. p. 217-218, e p. 235.

6. Ibidem, p. 205. Maravall si riferisce al Censo de personajes novelescos y legendarios, históricos y mythológicos, nombres geográficos y de autores citados por Cervantes en sus obras, collocato al termine delle Obras completas, a cura di Ángel Valbuena Prat, Madrid: Aguilar, $1960^{11}$.

7. Nell'interpretazione di Maravall, il Quijote rappresenta una peculiare modernità ispanica che conserva un atteggiamento di continuità e di nostalgia rispetto alle forme del tardo Medioevo, e che in un certo senso si sostanzia proprio attraverso l'estraneità alle idee di Machiavelli. Tale lettura emerge con piena (e forse, anche più marcata) evidenza nel saggio del 1948, El humanismo de las armas en don Quijote (del quale l'opera del '76, rappresenta una "nueva redacción»). Nel saggio del 1948, Maravall di fronte al medesimo passo cervantino che abbiamo proposto all'inizio si era limitato a indicare genericamente — senza affermare rapporti fra testi, neppure mediati- la penetrazione di «ingredientes de una técnica política de tono o mejor de nivel maquiavelico» (p. 247). 
Si sa che del Principe non erano disponibili edizioni in lingua spagnola. ${ }^{8}$ E d'altro canto noto che brani del Segretario fiorentino erano presenti in opere di scrittori spagnoli. Se andiamo all'origine della fortuna di Machiavelli in Spagna e guardiamo al Concejo i Consejeros del Príncipe di Furió Ceriol, la cui prima edizione compare ad Anversa nel 1559, possiamo anzi constatare proprio una eco della classificazione machiavelliana delle diverse forme di principato:

El cuarto tratado es en que se muestre al príncipe de reinar venciendo todas las dificultades [...]; y esto, por cuanto no se puede comprehender ni dar a entender sino por la variedad del reino o del principado en el cual se halla posesión en una de cuatro maneras, conviene a saber: ó por herencia, ó por elección, ó por fuerza, ó por maña; por tanto este tratado debe ser dividido en cuatro libros [...]. ${ }^{9}$

Però quelle righe di Furió Ceriol sono parte di una serie di proposte teoriche che intendono sintetizzare un progetto di lavoro (nel nostro caso si riferiscono al piano del IV trattato, poi mai redatto). E certamente quelle righe documentano un rapporto con Machiavelli - quelle come molte altre del Concejo - , però non ricalcano affatto la sua argomentazione, non entrano nel merito dei comportamenti del principe in relazione alla natura del principato. In definitiva, se si confrontano i tre testi — di Machiavelli, di Furió Ceriol, di Cervantes-, il passo del Quijote risulta senza dubbio più aderente all'originale fiorentino. Anzi ad essere più precisi, il passo cervantino è derivabile dall'originale di Machiavelli, ma proprio non è derivabile dal passo di Furió Ceriol.

A voler esercitare la memoria proprio in relazione a quello specifico passo, è anche noto che da un capitolo del Principe - lo ricordava molti anni fa proprio Maravall — ${ }^{10}$ procede la distinzione fra principati ereditari e principati conquistati che Álamos de Barrientos introduce nel Discurso al Rey del 1598: «Los reinos de vuestra Majestad se dividen en heredados y conquistados, y unos y otros en juntos, o apartados y divididos». ${ }^{11}$ Non risulta che esistano di tale discorso edizioni a stampa d'epoca ${ }^{12}$ e mi pare altamente improbabile che Cervantes avesse attinto ad un testo manoscritto. E tuttavia il confronto con quelle pagine risulta comunque istruttivo. Tutto il Discurso di Álamos —se si

8. Presso la Biblioteca Nazionale di Madrid esiste una traduzione manoscritta, che Helena Puigdomènech fa risalire alla fine del ' 500 (Helena PUigdomènech ForCADA, Maquiavelo en España. presencia de sus obras en los siglos XVI y XVII, Madrid: Fundación Universitaria Española, 1988, p. 117).

9. Fadrique Furió CERIOL, El Consejo y consejeros del Príncipe, in Curiosidades bibliográficas, colección escogida...por don Adolfo de Castro, Madrid: Biblioteca de Autores Españoles, 1907 , p. 319.

10. Cfr. José Antonio Maravall, "Maquiavelo y maquiavelismo en España», Boletín de la Real Academia de la Historia, 1969, p.182-218, poi in Estudios de historia del pensamiento espanol, vol. III, Madrid: Cultura Hispánica, 1984, p. 47.

11. Baltasar Álamos DE BARRIENTOS, Discurso político al rey Felipe III al comienzo de su reinado, a cura di Modesto SANTOS, Barcelona: Anthropos, 1990, p. 10.

12. Il Discurso di Álamos de Barrientos è pubblicato per la prima volta a Parigi nel 1867 , da Manuel Guardia con il titolo L'art de gouverner, discours adressé a Philippe III. 
vuole - ha sullo sfondo la classificazione machiavelliana, però, proprio perché fa sul serio, proprio perché è davvero orientato verso la funzionalità politica, si allontana quasi subito dal testo d'origine. In quanto è mosso dall'esigenza di usare davvero le categorie machiavelliane in rapporto a situazioni ben definite (gli immensi e discontinui territori dell'impero spagnolo), Álamos segue un percorso suo proprio: rispetto al passo sui principati nuovi acquisiti per grazia d'altri non argomenta mai con fedeltà tale da poter configurare una fonte intermedia della pagina cervantina. ${ }^{13}$ Sente anzi la necessità di introdurre subito un altro criterio complementare, quello della continuità o dicontinuità territoriale: juntos, o apartados y divididos.

Certamente, ci sono altri scrittori spagnoli che si richiamano a (o che richiamano) Machiavelli. Su uno di essi, Bartolomé Felippe, portoghese che scrive in spagnolo dando origine ad una storia editoriale che pare quasi un romanzo, ha richiamato recentemente e giustamente l'attenzione Juan Manuel Forte. ${ }^{14}$ Però neanche il libro di Felippe ha rilevanza rispetto al nostro scopo: cita sì diverse volte il nome di Machiavelli, anzi riproduce o riassume interi capitoli dei Discorsi, mostra diretta conoscenza delle Istorie Fiorentine, però né da segno di aver conoscenza del Principe, né tanto meno propone quella distinzione fra principati. Non è poi tanto facile trovare un intermediario tra Machiavelli e Cervantes che riproponga con un minimo di aderenza il testo originale in modo da poter costituire un ponte tra i due capolavori. ${ }^{15}$

13. L'argomentazione di Álamos si snoda in questa forma: «Los reinos de vuestra Majestad se dividen en heredados y conquistados, y unos y otros en juntos, o apartados y divididos. Conquistados llamo a aquellos que, aunque heredados y habidos legitimamente, han entrado por vía de guerra en esta corona, y contra la voluntad de los mismos naturales [...] y hago esta división porque de ella hemos de sacar qué diferente artificio y cuidado es necesario para los que son nuestros por fuerza y contra la voluntad de los naturales mismos, y que siempre están considerando el primer Estado que tuvieron, y para los otros, que con cualquiera diligencia y beneficio se conservan en aquel Estado a que están habituados [...] Sabemos que los conquistados siempre desean la restitución del primer Estado, hasta que del todo hayan faltado los que gozaron de él, y aún la memoria de ellos.» (Baltasar ÁLAMOS DE BARRIENTOS, Discurso politico al rey Felipe III al comienzo de su reinado, cit. p. 9). Manca assolutamente il tema dell'acquisizione per virtù o per grazia d'altri: ed è logico che manchi, perché mal si adatta al discorso sui possessi spagnoli da parte di un segretario del re.

14. Cfr. di Juan Manuel ForTe y Pablo Lopez ÁLVAREZ, l'«Introducción» al volume Juan Manuel FORTE (a cura di), Maquiavelo y España. Maquiavelismo y antimaquiavelismo en la cultura española de los siglos XVI y XVII, Madrid: Biblioteca Nueva, p. 26: «la obra del portugués Bartolomé Felippe merecería un tratamento diferenciado, pues Felippe no solo cita a Maquiavelo (los Discursos y la Historia de Florencia) como fuente de autoridad sin condena ni censuras [...], sino que hereda además muchas problemáticas genuinamente maquiavelianas: la dialéctica fines y medios, la diferencia entre leyes y ordenanzas, la renovación de los cuerpos mixtos. Estamos probablemente ante el más maquiaveliano de los tratados de la época escrito en español». Del libro di Bartolomé Felippe, ho potuto vedere, presso la biblioteca "Vittorio Emanuele» di Torino, soltanto la seconda edizione: Tractado del conseio y de los conseieros de los príncipes... Segunda impression, Turino [i.e. Londra!] Impresso en casa de Gio. uincenzo del Pernetto (i.e. John Wolfe!], 1589.

15. Quanto a Eugenio Narbona, si è forse esagerato sul «machiavellismo» della sua Doctrina politica civil escrita en aphorismos. Víctor Mínguez scrive addirittura che nell'edizione del 
Non pretendo con ciò di essere pervenuto a conclusioni inoppugnabili. Non ho la pretesa di aver esaurito tutte le possibili fonti di una conoscenza indiretta e mediata da parte di Cervantes. Si tratta di inventari che non sono mai completi; e poi si sa che passaggi di origine machiavelliana possono trovarsi inseriti nei luoghi più impensati. Mi pare tuttavia corretto sottolineare che il passo cervantino è a suo modo analitico ed incisivo, e contiene al suo interno quattro enunciati che rispondono fedelmente — nel significato e nella successione- all'originale machiavelliano:

- che i principati si distinguono in ereditati e acquisiti;

- che si entra in possesso di un principato acquisito o per propria virtù o per fortuna;

- che in questo secondo caso sono richieste doti specifiche per la sua conservazione;

— che laddove si è prodotta un'innovazione -e il principato nuovo lo è di per sé- è più probabile che ne vengano tentate altre nuove ed ulteriori.

È infine importante ricordare quanto ha sostenuto Helena Puigdmènech nel suo studio fondamentale: lo spettro limitato delle traduzioni a stampa (e la totale assenza per quanto riguarda Il Principe) non rappresentò una barriera insormontabile alla conoscenza di Machiavelli. ${ }^{16}$ Anche perché la barriera lin-

1604 — quella recogida per ordine dell'Inquisizione - Narbona «plagió descaradamente a Maquiavelo» («Introducción. De Dios y los reyes hispanos en la Edad Moderna», in Visiones de la monarquía hispánica, Castellò: Universitat Jaume I, Servicio de Publicaciones, 2007, p. 13). Lo studio scrupoloso e intelligente di Francisco Tomás y Valiente sulla documentazione inquisitoriale («La "Doctrina política civil” de Eugenio Narbona y la Inquisición», in Homenaje a José Antonio Maravall, Madrid: Centro de investigaciones sociológicas, 1985 , p. 405-416) consente una ricostruzione quasi completa del testo del 1604: troviamo passaggi che risalgono al Principe, e Machiavelli vi è nominato (come autor condenado), ma non pare proprio che emergano elementi da cui evincere lo "sfacciato" plagio da parte dello scrittore di Toledo. Quanto al nostro tema specifico - i principati acquisiti per grazia d'altri- non trovo proprio alcuna traccia che porti in tale direzione. Si può inoltre osservare che rispetto alla pubblicazione del primo volume del Quijote risulta comunque esclusa la stessa compatibilità cronologica. Le edizioni successive della Doctrina politica civil (Madrid,1621 e Madrid, 1799) richiamano la suma de privilegio per la stampa della prima edizione, e la datano al 9 ottobre 1604. Ma in quella data - secondo la ricostruzione di Francisco Rico- il testo del Quijote era già stato consegnato dall'autore, che nell'agosto lavorava alle parti preliminari: «la concentración en la piezas preliminares [en el mes de agosto 1604], supone que el testo del Quijote estaba ya en vías de publicación. Pocos o muchos meses atrás [...] Cervantes, pues, había presentado al Consejo de Castilla el original de la novela solicitando la licencia indispensable para imprimirla» (F. RICO, «Historia del texto", in Miguel de Cervantes, Don Quijote de la Mancha, cit., p. CCXXI e p. CCXXIII).

16. Cfr. Helena Puigdomènech Forcada, Maquiavelo en España. Presencia de sus obras en los siglos XVI y XVII, cit., p. 82: posto che la mancanza di traduzioni spagnole non trova sufficiente spiegazione né nella particolare efficacia della censura ecclesiastica, né tanto meno si deve a mancanza di interesse, non rimane che una risposta: «los lectores españoles de la época conocen la lengua italiana, que al fin y al cabo es la lengua de una parte, y muy importante, del imperio». 
guistica risultava alquanto attenuata dalla geografia politica del tempo che comportava tra Spagna e Italia una grande e intensa quantità di scambi e movimenti delle persone.

E tanto più tale considerazione dovrebbe valere nel caso nostro. Se si guarda alla straordinaria biografia di Cervantes - includendo in essa anche i gusti e le abitudini mentali- si danno tutte le condizioni e tutti i presupposti per una sua conoscenza più o meno diretta del Principe attraverso la lingua italiana. Si sa che verso la fine del 1569 Cervantes fugge in Italia per evitare una dura condanna - forse conseguente a un duello — che prevedeva il taglio della mano destra oltre a dieci anni di esilio. Nel 1570 Cervantes è a Roma, al servizio del cardinale Acquaviva. In veste di soldato scelto lo troviamo ancora in Italia negli anni 1574-75, a Genova, in Sardegna, a Napoli, a Palermo. S'imbarca a Napoli per rientrare in Spagna nel settembre del 1575.

$\mathrm{Al}$ fine di evitare sospetti infondati, tengo a dire che proprio non ho un passato né un presente nazionalista. Piuttosto, nella mia vita di studioso ho sempre tentato di combattere le storie delle letterature nazionali costruite per linee interne. La storia delle idee di quegli anni e dei secoli a venire non è né italiana né spagnola né francese, è una storia delle idee europea, ed è sul punto di diventare occidentale. Si sa che Cervantes, sia pure nel quadro del gioco narrativo, si attribuiva in fatto di letture una singolare inclinazione: "yo soy aficionado a leer aunque sean los papeles rotos de las calles». ${ }^{17} \mathrm{La}$ sua curiosità per i libri italiani è ben documentata, ed è davvero straordinario il modo con cui egli gioca con il lettore nella seconda parte del Quijote, al cap. LXII:

...Sucedió, pues, que yendo por una calle alzó los ojos don Quijote y vio escrito sobre una puerta, con letras muy grandes: "Aquí se imprimen libros », de lo que se contentó mucho, porque hasta entonces no había visto emprenta alguna y deseaba saber cómo fuese. [...] Llegó en esto a uno [oficial] y preguntóle qué era lo que hacía. El oficial le respondió:

- Señor, este caballero que aquí está —y enseñóle a un hombre de muy buen talle y parecer y de alguna gravedad - ha traducido un libro toscano en nuestra lengua castellana, y estoyle yo componiendo[...].

— ¿Qué título tiene el libro? — preguntó don Quijote.

A lo que el autor respondió:

- Señor, el libro, en toscano, se llama Le bagatele.

— ¿Y qué responde le bagatele en nuestro castellano? — preguntó don Quijote.

—Le bagatele — dijo el autor- es como si en castellano dijésemos "los juguetes»; y aunque este libro es en el nombre humilde, contiene y encierra en sí cosas muy buenas y sustanciales.

-Yo — dijo don Quijote- sé algún tanto del toscano y me precio de cantar algunas estancias del Ariosto. Pero dígame vuesa merced [...]: ¿̨ha hallado en su escritura alguna vez nombrar piñata?

—Sí, muchas veces — respondió el autor. ${ }^{18}$ 
E vero che ad oggi nessuno ha mai trovato un'opera italiana che rechi nel frontespizio il titolo Le bagattelle — «No se ha identificado ningún libro italiano titulado Le bagattelle», annota giustamente l'edizione diretta da Francisco Rico. ${ }^{19}$ Però è anche vero che quella pagina rivela in fatto di scrittori italiani non solo una straordinaria curiosità intellettuale, ma anche in un certo senso una competenza specifica. In questa circostanza la mente va a quegli scritti che, utilizzando un tono mordace e faceto, dicono cose assai serie - que "contiene [n] y encierra [n] en si cosas muy buenas y sustanciales». "Bagatelle» è un termine che ricorre negli scritti orientati in senso ironico: Machiavelli usa la parola, ma, che io sappia, non negli scritti scientifici bensì in un testo teatrale, La Clizia, che viene poi presentata nel frontespizio della prima edizione a noi nota nella veste di commedia facetissima [...] composta per lo ingenioso huomo Nicolò Machiavelli fiorentino. ${ }^{20}$ I termini «bagattelle» e "pignatte» occorrono per esempio in un libro che è certo tra le fonti della tradizione di quel Cinquecento capriccioso e irregolare dei lettori di Luciano ed Erasmo di Aretino e Doni e di altri peregrini ingegni. ${ }^{21}$ Troviamo insieme quei termini ne I dilettevoli dialogi, le vere narrationi, le facete epistole di Luciano filosofo, ${ }^{22}$ vale a dire nella traduzione di trenta testi di Luciano, ad opera di quel Niccolò Leoniceno che Ariosto ricorda nel canto XLVI dell' Orlando Furioso: scrittore oggi si direbbe "padano" in considerazione del luogo della sua nascita, ma che s'ingegna di tradurre nella lingua «toschana», come vanta Nicolò Zoppino editore del libro:

Legge adunque o lettor mio tal opera novamente in luce venuta [...] et quando conoscero le fatiche nostre in qualche parte esserne satisfacevoli et grate, daremo opra che di maggior cosa gli huomini volgari per me partecipi siano, et per che la lingua toschana a tempi nostri non solamente appresso di quelli che le latine lettere non sanno, ma etiamdio appresso de huomini literatissimi in maggior precio et reputatione che mai la fosse, essere si vede, sforzaromi in cio che per l'avenire l'opre nostre et per la nuova ortographia, et per la semplice lingua toschana grate et laudevoli appresso di ciascuno lettore si restino, non mi curando di spesa, ne di faticha alcuna che gire vi potesse $[\ldots]^{23}$

19. Ivi.

20. Così nel frontespizio dell'edizione fiorentina del 1537. Cfr. Atto III, Scena, II. Sul termine «bagattelle» richiama l'attenzione Luigi Blasucci (curatore del IV volume delle Opere, Scritti letterari), annotando che il termine fiorentino sta per "giuochi di mano fatti dai giocolieri» (Torino: Utet, 1989, p 193). Il che forse spiega perché Cervantes traduca la parola toscana «bagattelle» con quella castigliana «juguetes».

21. È il titolo del saggio di Paolo Procaccioli che apre il volume, Cinquecento capriccioso e irregolare. Eresie letterarie nell'Italia del classicismo, a cura di Paolo Procaccioli e Angelo Romano, Roma: Vecchiarelli, 1998.

22. Nicolò DA LONIGO, I dilettevoli dialogi, le vere narrationi, le facete epistole di Luciano filosofo, Biblioteca italiana, 2005, in www.bibliotecaitaliana.it (Stampato in Vinegia per Nicolo di Aristotile detto Zoppino, Nel'anno del Signore. MDXXV) Il vocabolo «bagatelle» (così scritto) compare una volta nel dialogo 2 (p. 15); il vocabolo "pignatte» compare tre volte, rispettivamente nei dialoghi 1, (p. 12), 2 (p. 17), 6 (p. 37).

23. Ibidem, p. 1. 
Anche se negli anni della redazione del Quijote sono passati decenni dal periodo italiano, non credo che sussistano dubbi sulla capacità, sulla curiosità e sulla frequentazione di Cervantes verso opere italiane. Il gusto di misurarsi con la lingua italiana lo ritroviamo fra l'altro anche nelle Novelas ejemplares, precisamente nel Licenciado Vidriera, dove lo scrittore prima sciorina, sia pure storpiati, una serie di motti italiani da osteria («pasa acá, manigoldo; venga la macarela, li polastri e li macarroni»), poi si cimenta in una rassegna enologica delle terre d'Italia, ed evoca tra l'altro il vino "Cinque Terre» — "la grandeza del de las Cinco Viñas». ${ }^{24}$ Non mi pare proprio che né la biografia, né gli scritti di Cervantes portino ad escludere l'ipotesi di una lettura diretta del Princi$p e .{ }^{25}$ Semmai inducono a considerarla non solo possibile, ma anche molto probabile. Pensare a una recezione ingenua e inconsapevole della fonte mi pare ipotesi che alla fine offende la stessa scaltrezza cervantina, anche perché - come potrà vedersi in seguito- quel passo trascritto all'inizio non costituisce una circostanza del tutto episodica ed isolata rispetto a tematiche riconducibili all'ampio bacino delle discussioni del tempo su Machiavelli e sul machiavellismo.

Detto ciò, esiste naturalmente un altro piano della riflessione. Che senso ha, nel quadro della figura intellettuale di Cervantes, l'ascendenza machiavelliana di quell'insegnamento che Quijote impartisce a Sancho? Certo, il passaggio che ho richiamato è di per sé un invito a prendere le distanze da interpretazioni cervantine magari affascinanti, ma molto costruite e ideologicamente calcate. Penso per esempio alla tesi che Fernando Rielo propone senza possibilità di sfumature:

La oposición de los dos personajes, literario e histórico, encierra el antagonismo en que se ha movido desde siempre la sociedad humana: utopía e historia [...] Don Quijote encarna la utopía y Maquiavelo la historia. [...] En Don Quijote, la eternidad, en Maquiavelo la temporalidad [...] una ética y una política. ${ }^{26}$

Francamente credo che Cervantes avesse una testa un po' più complicata (e certamente più ironica) di quanto si può evincere da interpretazioni di questo tipo.

24. Miguel de Cervantes, Novelas ejemplares, edición de Florencio Sevilla Arroyo y Antonio Rey HaZAS, Madrid: Espasa Calpe, 1995, vol. I, p. 305.

25. Manuel Fernández Álvarez non si pronuncia circa la lettura del Principe da parte di Cervantes, anche se si esprime in modo tale da collocarla fra gli oggetti di attrazione intellettuale: «[...] eran los hombres de Letras, los humanistas y escritores, los que fascinaban al joven poeta. Desde aquellas cumbres de la poesía de la Baja Edad Media (el Dante, Petrarca, Boccaccio) desde el magisterio de los egregios humanistas (Marsilio Ficino, Pico della Mirandola), hasta los nuevos valores que deslumbraban en el quinientos: Maquiavelo con su Principe, leído por tantos y practicado por muchos más, aun sin leerlo [...]» (Cervantes visto por un historiador, Madrid: Espasa Calpe, 2005, p. 80).

26. Fernando Rielo, Teoría del Quijote. Su mística hispánica, Madrid: Ediciones José Porrúa Turanzas, 1982, p. 110. 
Comprendo d'altra parte che la presenza di questa traccia machiavelliana segnata dal passo sui principati acquisiti non per propria virtù, ma per grazia di altri possa ingenerare, almeno a una prima occhiata, una certa sorpresa sulla quale merita riflettere. Ma come — vien da dire_ l'ascendenza di Cervantes, pur trasfigurata dal tempo e dalla stessa sua grandezza, non è erasmiana? E come possono andare insieme Erasmo e Machiavelli: da un lato un uomo religioso (anche se critico e corrosivo) che si adopera per la pace e per la riforma interiore e dei costumi; dall'altro un esponente forte e radicale del Rinascimento non cristiano, impegnato in più luoghi a dimostrare la centralità della guerra come premessa della formazione di autentici valori civili. In realtà non è così ovvio che nella concretezza della storia delle idee Erasmo e Machiavelli non possano andare insieme. Su questo punto il caso della Spagna è davvero emblematico: il primo degli antimachiavellici — Juan Ginés de Sepúlvedaè ad un tempo e prima di tutto un feroce avversario di Erasmo. All'opposto, il primo degli intellettuali considerati machiavellici, Furió Ceriol, l'autore del Concejo, è prima autore del Bononia, ${ }^{27}$ un'opera che in qualche modo radicalizza posizioni erasmiane. Sono combinazioni che non sono accidentali, ma hanno una loro ragion d'essere. Sepúlveda è il portatore di un cristianesimo non solo militante, ma militare, e dunque è avversario di Erasmo — che ha posizioni ireniche e avverse alla guerra —, però è d'altro canto avverso a Machiavelli, che ha sostenuto nei Discorsi una tesi «empia»: che il Cristianesimo ha prodotto uomini inetti ai valori civili e alle virtù guerriere. Quanto alla presenza di Erasmo e Machiavelli in Furió Ceriol, bene la illustra Luca D’Ascia:

[Ceriol si proponeva] di prendere da Erasmo e da Machiavelli quanto poteva servire ad una politica nazionale spagnola, nutrita di valori civili e anche militari, ma priva di rigidità controriformistiche, guidata da laici religiosamente consapevoli (perché lettori della Bibbia) anziché da religiosi intransigenti pronti a subordinare il merito alla conformità. ${ }^{28}$

A proposito di quella traccia machiavelliana nel Quijote, credo che il tema vada trattato con sobrietà. Quel passo fa di Cervantes un lettore di Machiavelli, ma non ne fa un «machiavellico», anche perché le pagine del Fiorentino possono essere apprezzate per motivi vari e diversi: non solo come fonte di precetti politici, ma anche per la disincantata e beffarda descrizione del mondo com'è. Non credo invece che quel passaggio tra i due capolavori sia accidentale e privo di significato. In realtà intellettuali come Erasmo, Machiavelli, Cervantes hanno in comune un modo di leggere il mondo, che si lascia alle spalle un passato e un universo intellettuale. Prima di tutto hanno alle spalle una negazione: non sono aristotelici. Per nessuno dei tre l'essere, in quan-

27. Fadrique FURIÓ CERIOL, Bononia, sive de libris sacris in vernaculas linguas traducendis libri duo, Basileae: Oporinus, 1556.

28. Fadrique FurIó CERIOL, Il Consiglio e i consiglieri del Principe, a cura di Luca D’AsCIA, Roma: Bulzoni, 2007. Cfr. l'«Introduzione» di Luca D’AsCIA, p. 10. 
to è e come è, anche è dotato di valore sulla base dell'idea che ogni cosa contiene in sé il principio del suo sviluppo (nel quadro di un universo sensato e gerarchicamente ben ordinato). Questi intellettuali non leggono le cose del mondo attraverso il filtro-apparato della Scolastica. La realtà urge ed incombe con la sua pressione, con la forza delle sue contraddizioni. Gli strumenti di lettura tratti dalla cultura esistono ma non sono rigidi, e devono essere riattualizzati. Sono piuttosto attrezzi per meglio pensare: sono le storie dei classici (in Machiavelli), la Scrittura filtrata attraverso l'umanesimo filosofico (in Erasmo), la stessa tradizione erasmiana tuttavia arricchita da una vasta letteratura a lui coeva (Cervantes). Quello che proprio non c'è in questi intellettuali (benché proprio questo Avalle ${ }^{29}$ abbia pensato di veder affermato più tardi nel Persiles) è la "grande catena dell' essere». L'essere, anzi, non è propriamente essere, la realtà è aporetica. Sono intellettuali che guardano alla realtà senza cadere nell' "incanto», che mantengono ferme le distinzioni, i punti di vista, i diversi pareri, le contraddizioni del reale, che non si accomoda a comporre un unico, armonico, grande disegno. ${ }^{30}$ Prima di tutto in questa forma sono davvero ed effettivamente moderni. Erasmo, Machiavelli, Cervantes hanno interessi e idee alquanto diversi, però tutti e tre elaborano strategie per guardare le cose del mondo dopo aver creato una distanza mentale che li sottrae tanto al pensare comune - «el antiguo legislador que llaman vulgo»_ 31 quanto alle dottrine ufficiali.

Sotto questo profilo non è strano che Machiavelli abbia lasciato un segno anche nell'autore del Quijote, e sono convinto che quel passo attesti senz'altro una curiosità intellettuale verso l'analisi del potere e delle sue forme compiuta dal Fiorentino. Non cadeva certo al di fuori degli interessi di Cervantes una lettura secolarizzata della fattualità delle relazioni umane. Non c'è dubbio sulla fede cristiana di Cervantes, però anche questa è sui generis: l'incombere dell' $i$ stituzione ecclesiastica e dei suoi bracci, del suo sistema dottrinale di regole e imperativi cui si deve obbedienza, si avverte tante volte. Però nel dipanarsi della novela e nella costruzione dei personaggi emerge in primo piano e con carattere di marcata autonomia la dimensione della scelta individuale sorretta da una forte tensione etico-religiosa. Esiste una relazione sottile, ma profonda, tra una religiosità che concede al credente un ampio spazio di vissuto personale e l'esigenza di una lettura secolare della politica: nell'uno e nell'altro livello — nella religione come nella politica — vengono a restringersi gli spazi del sacerdotalismo. A partire da ciò Machiavelli rappresenta l'altezza dei tempi, costituisce la linea che segna un confine: anche se poi, muovendo da quel con-

29. Cfr. Miguel de Cervantes, Los trabajos de Persiles y Sigismunda, edición, introducción y notas de Juan Bautista Avalle-ArCE, Madrid: Editorial Castalia, p. 20. Avalle si richiama all'Essay on man di Alexander Pope (1733).

30. Rafael Del Áquila ci ha lasciato un bellissimo saggio su "Tragedia e ironía en la teoría política de Maquiavelo", (Ingenium. Revista de historia del pensamento moderno, n. 2, juliodiciembre 2009, p. 3-23), dove descrive la funzione dell'ironia nell'originalità del pensare machiavelliano (p. 15).

31. Miguel de Cervantes, Don Quijote de la Mancha, cit., p.11. 
fine, le risposte sono ogni volta diverse. Diceva recentemente Enzo Baldini che ogni generazione ha costruito il suo Machiavelli. ${ }^{32} \mathrm{Ed}$ è proprio così: se da un lato una lettura fattuale e secolarizzata della politica, può anche essere avversata, ma è destinata a restare nel lungo periodo, contaminando nel profondo gli stessi avversari, dall'altro lato i termini e le configurazioni di potere entro cui si costruisce e si propone l'agire politico mutano continuamente insieme all'orizzonte stesso dei possibili. In fondo questi termini sono già altri alla data della pubblicazione delle opere maggiori di Machiavelli, e saranno radicalmente cambiati all'inizio del ' 600 .

Se poi — pur nel quadro di questo segno comune della prima, lunga età moderna-, spingiamo la comparazione e lavoriamo di cesello, allora troviamo che, anche quando esiste una radice comune - la secolarizzazione che avanza-, le piante che nascono sono diverse.

Proviamo a cercare nei complessi percorsi mentali del Quijote una linea di sviluppo a partire da quella traccia machiavelliana che abbiamo annotato. Ebbene, c’è nel Quijote un ampio passaggio - l'unico - dove si enuncia il «consequenzialismo", s'insiste sulla correlazione mezzi-fini e sull'uso spregiudicato dei mezzi. Ad esprimersi in tal senso è per giunta proprio Quijote, vale a dire l'incarnazione dell'irresponsabilità, così come emerge tante volte nell'opera, ma in particolare a proposito della sfortunato epilogo della vicenda di Andrés. L'esito della solerzia cavalleresca di Quijote è stato disastroso: il giovane ne ha ricavato una dose doppia e rinnovata di frustate, tante da lasciarlo quasi morto. E tuttavia l'hidalgo si preoccupa prima di tutto della sua aderenza alle norme: egli aveva agito secondo un nobile scopo, si era comportato secondo le regole della cavalleria, aveva provveduto a giurare e a far giurare sul rispetto degli impegni.

Ogni discussione intorno al rapporto tra i mezzi e i fini sembrerebbe pertanto esclusa (perché risolta in partenza), e tanto meno una riconsiderazione della legittimitazione dei primi alla luce dei secondi, argomento di cui peraltro all'epoca si faceva un gran parlare, in chiave ora machiavellica ora antimachiavellica, secondo complicate casistiche.

E invece c'è una pagina in cui Quijote propone il tema, esaltando anzi, questa volta, l'uso spregiudicato dei mezzi. Però — qui sta il punto- nell'avanzare tale eccezione, l'esaltazione dell'uso spregiudicato dei mezzi non riguarda lo stato e la vita pubblica. Riguarda la vita amorosa. Basilio si è finto ferito a morte per ottenere la mano di Quiteria che era destinata a Camacho el Rico... che avrebbe così sposato una vedova (però beninteso integra). Il fatto è che Basilio non è affatto in punto di morte, e pretende assumere come valido il vincolo coniugale che considera stabilito attraverso la finzione della imminente morte per amore. ${ }^{33}$ Così commenta Quijote difendendo Basilio, secondo una prospettiva nient' affatto in linea con i precetti tridentini:

32. Nella relazione di chiusura al recente convegno in memoria di Luigi FIRPO, Machiavellismo, Platonismo ed eterodossia culturale in Italia nell'età moderna, Torino, 25-26 settembre 2009.

33. Cfr. sul tema il brillante saggio di Alessandro MARTinENGO, «La novella di Basilio e Quiteria», in "I mondi possibili del Chisciotte», Critica del testo, IX, 2006, 1-2, p. 119-133. 
Teneos, señores, teneos ... y advertid que el amor y la guerra son una misma cosa, y asì como en la guerra es cosa lícita y acustumbrada usar de ardides y estratagemas para vencer al enemigo, asì en las contiendas y competencias amorosas se tienen por buenos los embustes y marañas que se hacen para conseguir el fin que se desea, como no sean en menoscabo y deshonra de la cosa amada. ${ }^{34}$

Quijote conclude e sintetizza che «no se pueden ni deben llamar engaños [...] los que ponen la mira en virtuosos fines.Y que el de casarse los enamorados era el fin de más excelencia». ${ }^{35}$

Sembra quasi che Cervantes stia parodiando Machiavelli, o se vogliamo la vulgata machiavellica, che si stia prendendo gioco del lettore ignaro, oppure che strizzi l'occhio a quello sapiente, chiamandolo ad una qualche forma di divertita complicità, che suona ad un tempo come irrisione alla dotta magniloquenza ostentata dal personaggio Quijote. ${ }^{36}$ Quello che a me pare escluso è che si tratti semplicemente, di un pour parler, che non si tratti di una scrittura dotta e consapevole, che Cervantes non sappia di giocare con questioni che attengono a Machiavelli, o alla vulgata che circola nel suo tempo.

Cervantes è senza dubbio uno scrittore cristiano ed è uno scrittore prudente, ma non è uno scrittore innocente. Ci sono passaggi nella sua opera, che risultano alquanto strani ad una lettura piana. Maurice Molho ${ }^{37}$ aveva notato curiose ambiguità nel Coloquio de los perros. Proprio in quell'opera, il nostro geniale scrittore si è appena espresso sui Gesuiti con parole di grande elogio - sono i buoni Padri che insegnano ai giovani-. Adesso improvvisamente, pochi capoversi di seguito, tira fuori la «razón de estado» e la personifica in una "señora». Però, in verità, siamo in un luogo del racconto dove la ragion di stato, per quanto attiene alla vicenda di Berganza, proprio non c'entra per nulla:

Desta gloria y desta quietud me vino a quitar una señora que [...] llaman por ahí razón de estado; que, cuando con ella se cumple, se ha de descumplir con otras razones muchas. Es el caso que aquellos señores maestros - [es decir los padres de la Compañia de Jesus] — les pareció que la media hora que hay de lición a lición la ocupaban los estudiantes, no en repasar las liciones, sino en holgarse conmigo; y así, ordenaron a mis amos que no me llevasen más al estudio. $^{38}$

34. Miguel de Cervantes, Don Quijote de la Mancha, cit., p. 880-881.

35. Ibidem, p. 883.

36. Circa i giochi di trasfigurazione e di sottile esasperazione ironica che si consumano nel Quijote attraverso il percorso fonte-personaggio-lettore ha fornito raffinate e acute analisi Aldo Ruffinatto, a proposito del rapporto con Ariosto: «Wi R Wj: relaciones de accesibilidad (R) entre el mundo de don Quijote (Wj) y el mundo de Orlando (Wi)», in "I mondi possibili del Chisciotte», Critica del testo, IX, 2006, 1-2, p. 239-253.

37. Cfr., di Maurice Molho, i Remarques introduttivi all'edizione bilingue de Le mariage trompeur et colloque des chiens, Paris: Aubier-Flammarion, 1970, p. 38-39.

38. El coloquio de los perros, in Novelas ejemplares, cit., vol. II, p. 580. 
In verità i religiosi hanno solo detto ai padroncini di Berganza di tenerlo a casa, perché distrae gli alunni dagli studi. C'è un venir meno del senso in questi passaggi, c'è davvero un salto logico. Però alla fine, in base alla narrazione, risulta oggettivamente vero che sono i buoni padri quelli che fanno uso di quella "señora", vale e dire della ragion di stato. Non si capisce bene chi e quanti prenda in giro Cervantes: è un modo (certo non il più semplice e piano) per schierarsi contro la ragion di stato? sta ironizzando sul fatto che se ne parla continuamente, a proposito e a sproposito? intende insinuare che i Gesuiti sono maestri nell'uso della ragion di stato?...

Peraltro il modo di affrontare il tema della ragion di stato in due righe è scherzoso e faceto ma è anche acuto, penetrante. Perché il problema sta proprio lì: la ragione dello stato è in conflitto con mille altre ragioni, motivazioni, valori, che esistono nel vivere degli uomini. La morale di Cervantes - almeno per quel che attiene alla libertà di giudizio, allo sguardo interiore della coscienzanon era moralistica, la sua intelligenza era sovrana e tale da non rinunciare del tutto a qualche gioco ardito.

Ritorniamo ora a riproporre in forma sintetica e conclusiva la questione della prossimità e della distanza fra Cervantes e Machiavelli. Lo scrittore spagnolo appartiene ad un mondo che Machiavelli ha tanto contribuito a promuovere, dove:

- tutti i valori rimangono vitalmente e conflittualmente collocati sullo stesso tavolo e non si danno facili ricomposizioni;

- l'autorealizzazione è tanta parte del senso del vivere, al punto che dove si danno vincoli troppo stretti, dove l'orizzonte delle opportunità è troppo limitato, vengono sminuiti il senso, la dignità, il valore della vita.

Tuttavia questo individualismo e questo senso della libertà hanno subìto col tempo, dagli anni di Machiavelli, una radicale trasfigurazione. La distanza tra i due non discende dal fatto che Cervantes sia programmaticamente orientato contro le idee del Fiorentino in virtù di un'opposizione moralistica che attinge alla precettistica delle autorità del suo tempo. Quella distanza ha ben altra origine: i contenuti che avanzano ad occupare il primo piano della scena cervantina sono la passione amorosa, la vocazione professionale, le credenze religiose. Essi prefigurano un intimismo della libertà che segna il primato del privato sul pubblico. Il dolore del padre di Zoraide, l'imperativo di lasciare ai figli le scelta della professione e quella del compagno o della compagna di vita; la descrizione del rinnegato che è rinnegato ma che non è malvagio, il dramma di Ricote a mezzo tra due mondi che le istituzioni non consentono di comporre, l'ambiguo accenno alla libertà di coscienza: sono tutti luoghi questi, che attraverso la straordinaria leggerezza cervantina rinviano alle profondità dell'anima, a quel profondo che la precettistica esteriore non può governare. E per questi aspetti, con il suo intimismo, con la sua carica specifica di problematicità il Quijote rinvia ad un'epoca ben posteriore al Principe di Machiavelli, fa pensare piuttosto ad un'opera cronologicamente assai più vicina, e 
anch'essa straordinaria ed eccelsa nel suo genere: agli Essais di Montaigne. ${ }^{39}$ $\grave{\mathrm{E}}$ tanto facile quanto immediato osservare che rispetto a Montaigne il punto di vista di Cervantes è molto spesso contiguo: nel Coloquio de los perros fa pensare a Montaigne l'idea dell'animale che si rende giudice scandalizzato, discettando da un superiore punto di vista sul supponente mondo degli uomini. Nello stesso dialogo fa ancora pensare a Montaigne l'idea che tra gli uomini "pocas o ninguna vez se cumple con la ambición que no sea con daño de tercero". ${ }^{40}$

I ripetuti rimandi e raffronti con Montaigne che troviamo in El pensamiento de Cervantes di Américo Castro ${ }^{41}$ possono essere discussi e discutibili nel dettaglio, ma colgono una prossimità mentale innegabile. Sull'argomento, in tempi più recenti Otilia López Fanego ha scritto un saggio tanto cauto e modesto nel suo titolo, quanto analitico e insieme profondo nella sostanza. Le analogie riguardano una quantità impressionante di temi: "Alma y cuerpo; incostancia de la naturaleza humana; virtud y gloria; imaginación, encantos, profecías; Religión, riqueza y pobreza; virtud y nobleza....actitud antidogmática, relatividad de los juicios humanos, espiritu tolerante y abierto". ${ }^{42}$ Sono persuaso che tale prossimità mentale — qualunque sia la sua origine- ${ }^{43}$ risieda prima di tutto in una modernità attraversata dal disincanto, una modernità che non conosce più le certezze antropocentriche del Rinascimento, e che si avvia ad abbandonare il primato della sfera pubblica, ormai lavorando alla costruzione dell'interiorità privata.

Proprio mettendoci in quest'ottica possiamo misurare la prossimità e la distanza tra Machiavelli e Cervantes. Sono entrambi due campioni della libertà, amano la libertà, la esaltano: però, attenzione, solo fino ad un certo punto

39. Per il versante francese di questa grande svolta che si compie nella cultura europea rimane fondamentale il libro di Annamaria BATTISTA, Montaigne e Charron. Alle origini del pensiero politico libertino, Milano: Giuffrè, 1979.

40. El casamiento engañoso y el coloquio de los perros, in Novelas ejemplares, cit., vol. II, p. 577. Cfr. Michel de Montaigne, Essais, I, XXII: Le profit de l'un est dommage de l'autre (Oeuvres complètes, Paris: Gallimard, 1962, p. 105 e sgg.).

41. Cfr. Américo Castro, Il pensiero di Cervantes, a cura di Marco Cipolloni, Napoli: Guida, 1991 , in particolare il cap. 7 , dedicato alla morale.

42. Otilia López FANEgo, "Contribución al estudio comparado de Montaigne y Cervantes», Anales Cervantinos, 1979-1980, p. 163-207, in part. p. 164-165.

43. Otilia López Fanego si richiama alla filosofia della storia di Hegel e fa risalire alla comune radice epocale il fatto che tra Montaigne e Cervantes «existan sorprendentes parecidos —independientes de influencias personales directas...» (cfr. ivi, p. 163). Però, se è vero che non disponiamo di accostamenti testuali tali da provare la lettura di Montaigne da parte di Cervantes, neppure siamo in grado di negarla in modo assoluto. E' vero che non conosciamo un Cervantes lettore di libri francesi, e che la prima traduzione spagnola degli Essais è posteriore alla sua morte. Tuttavia esisteva la traduzione italiana (pur limitata ad alcuni capitoli) dei primi due libri degli Essais condotta sull'edizione francese del 1580: Discorsi morali, politici, et militari del Sig. Michiel di Montagna... tradotti dal Sig. Girolamo Naselli dalla lingua Francese nell'Italiana. Con un discorso se il forastiero si deve admettere alla administratione della Repubblica, Ferrara: per Benedetto Mamarello, 1590. Di tale traduzione esiste una copia alla Biblioteca Nazionale di Madrid. 
trattano e parlano della stessa libertà. C'è in Cervantes come c'era in Machiavelli l'elogio della libertà come autonomia, come condizione che non ci pone in obbligazione con alcuno. Così nel famoso passo della parte seconda, al cap. LVIII:

La libertad, Sancho, es uno de los más preciosos dones que a los hombres dieron los cielos; con ella no pueden igualarse los tesoros que encierra la tierra ni el mar encubre; por la libertad, así como por la honra, se puede y debe aventurar la vida, $y$, por el contrario, el cautiverio es el mayor mal que puede venir a los hombres. Digo esto, Sancho, porque bien has visto el regalo, la abundancia que en este castillo que dejamos hemos tenido; pues en metad de aquellos banquetes sazonados y de aquellas bebidas de nieve me parecía a mí que estaba metido entre las estrechezas de la hambre, porque no lo gozaba con la libertad que lo gozara si fueran míos, que las obligaciones de las recompensas de los beneficios y mercedes recebidas son ataduras que no dejan campear al ánimo libre. ¡Venturoso aquel a quien el cielo dio un pedazo de pan sin que le quede obligación de agradecerlo a otro que al mismo cielo!

Anche il Machiavelli repubblicano insiste sulla condizione di autonomia personale, che infatti fa dei Romani, anche quando plebei, dei soggetti capaci di giocare un ruolo attivo nella vita pubblica. Però quell'autonomia s'inscrive in Machiavelli nella cornice del primato dell'uomo pubblico, ${ }^{44}$ nello spazio della gloria, della grandezza civica, dell'impresa politica; al contrario in Cervantes prevale la dimensione appartata e privata, entro un orizzonte prospettico che prende anzi una discreta distanza rispetto alle manie e ai deliri di grandezza della politica.

Se mettiamo insieme i tasselli dei diversi luoghi del Quijote che abbiamo preso in esame - quelli che hanno rapporto o con Machiavelli o con tematiche che sono state a lui ricondotte- troviamo sempre in puntuale corrispondenza un distacco e un gioco ironico che fanno da contrappunto a quelle presenze.

In relazione al passo citato all'inizio, al tono erudito e saccente con cui Quijote introduce i modelli di comportamento da adottarsi nei principati nuovi acquisiti per grazia d'altri, segue il comico e del tutto impolitico tornare al segno di Sancho che richiama subito alle botte prese e al corpo dolorante: «En este que ahora nos ha acontecido [...] quisiera yo tener este

44. È vero che in Machiavelli gli ordinamenti liberi sostengono la dedizione al bene pubblico, ma anche la ricchezza privata e l'espansione demografica (Cfr. per esempio l'intero capitolo II del libro II dei Discorsi). Tuttavia non credo che in Machiavelli la libertà negativa e la libertà politica stiano precisamente nel rapporto delineato da Quentin Skinner: "...la ragione che Machiavelli ci offre per coltivare le virtù e servire il bene comune... è sempre che tali azioni rappresentano, in realtà, il migliore e l'unico mezzo per assicurarci una qualche misura di libertà individuale per perseguire gli scopi che ci siamo prefissi» (Quentin SKInNER, Virtù rinascimentali, Bologna: Il Mulino, 2006, p. 270). Almeno non è vero che la libertà politica è strumentale alla possibilità di operare in vista del benessere privato. 
entendimiento y este valor que vuestra merced dice; mas yo le juro, a fe de pobre hombre, que más estoy para bizmas que para pláticas». ${ }^{45}$

Poi nel secondo volume vien fuori che si può derogare alle regole della morale corrente e persino ai precetti ecclesiastici, però beninteso soltanto quando è in gioco la finalità più alta, suprema...vale a dire far sì che si compiano i desideri d'amore.

Infine, nel Coloquio de los perros, della gran señora - la ragion di statofanno uso i buoni padri Gesuiti per allontanare il cane Berganza che distrae gli allievi dall'applicazione allo studio.

Otteniamo un risultato convergente se proviamo a compilare un elenco delle azioni effettivamente ben riuscite nel capolavoro cervantino. Riguardano l'amore (Luscinda, Dorotea, Quiteria...), la libertà in senso stretto (la fuga da Algeri), la fede interiore (Zoraide), l'amicizia (Sancho non denuncia Ricote, anche se non gli presta aiuto per dissotterrare il suo tesoro), e infine ...la stessa «buona morte» di Quijote, che si compie senza retorica in un quadro di sobria serenità: tutte azioni appartate rispetto alla dimensione della vita pubblica. Non è che Cervantes contrapponga alla politica di Machiavelli un'altra politica radicata nella trascendenza e fondata sulla teologia. E' vero piuttosto ch'egli colloca la politica in un discreto secondo piano, non la considera in senso forte un luogo eminente dell'autorealizzazione dell'uomo. E anzi, i temi della politica — come di tanti altri aspetti del vivere - si trasformano in attrezzi con cui si destreggia la sua divertita ironia. 